\title{
The Relationship Between "Jiubian" and "Jiuge" and the Inheritance by Qu Yuan and Song Yu
}

\author{
Kunfen Bai \\ The College of Literature and Journalism \\ Sichuan University \\ Chengdu, China 610065
}

\begin{abstract}
Whether Song Yu was a disciple or student of Qu Yuan has become a mystery since the Han Dynasty. According to Sima Qian's "Records of the Historian - Biography of Qu Yuan and Jia Yi", "after the death of Qu Yuan, there were Song Yu, Tang Le and Jing Chai in Chu. All of them were good at literature, by which they were known by people. They all followed the euphemistic writing style of Qu Yuan, and always dared not advise directly to the monarch." It is said that Song $\mathrm{Yu}$ was the first person known by his $\mathrm{Fu}$ after $\mathrm{Qu}$ Yuan's death. However, Wang Yi, who was only one hundred years away from Sima Qian, said in "Chuci Zhangjiu Jiubian": "Song Yu, Qu Yuan's disciple, also showed compassion for his teacher, loyal but exiled, wrote 'Jiubian' to describe the aspirations of his teacher." Wang Yi said Song Yu was a disciple of the disciple of Qu Yuan. Sima Qian and Wang Yi said differently. Therefore, it caused a dispute whether Song Yu was a disciple or a student of Qu Yuan. Based on the text analysis of Song Yu's "Jiubian" and Qu Yuan's "Jiuge", this paper explores the relationship between the content and style of the two works, and combs the clues of this problem.
\end{abstract}

Keywords—“Wenxindiaolong · Biansao"; "Jiubian"; “Jiuge”; Qu Yuan; Song Yu

\section{INTRODUCTION}

"Wenxindiaolong - Biansao" is Liu Xie's special treatise on $\mathrm{Chu} \mathrm{Ci}$, involving comments related to Song Yu's works, such as: "Jiubian" and "Jiuge" are very beautiful but sad." Wang Yi's "Chuci Zhangju" has made it clear that "Jiubian" was "made by Song Yu, the official of Chu". ${ }^{1}$ Liu Xie put Qu Yuan's "Jiubian" and Song Yu's "Jiuge" together, which is easy to point out a comparative perspective, that is, Song Yu's inheritance of Qu Yuan. Song Yu's "Jiubian" is marked as "Jiubian and Wuge" in Volume 33 of "Wenxuan", and Qu Yuan's "Jiuge Ershou" and "Jiuzhang Yishou" are in the same volume. It shows that the nine paragraphs in "Jiubian" can be written independently. Song $\mathrm{Yu}$ has always been regarded as Qu Yuan's disciple. In Wang Yi's "Preface to Jiubian", he said: "Song Yu, a disciple of Qu Yuan, also showed compassion for his teacher, loyal but exiled, wrote 'Jiubian' to describe the aspirations of his teacher." $\mathrm{Li}$ Shanzhu copied this paragraph. According to common sense, students will inherit the images and expressions in teachers'

See Hong Xingzu's "Supplementary Notes to Chu Ci”, Zhonghua Book Company, 1983, P 183. works. Did Liu Xie pay attention to the inheritance relationship between "Jiubian" and "Jiuge"? Does this relationship exist? This chapter is intended to investigate through text analysis.

\section{SONG YU's MOURNING ON AUTUMN}

Song Yu's "Jiubian" is most praised for the theme of "mourning on autumn". The first, third and seventh chapters are all written about autumn. The first chapter is especially recited by later writers.

The rustle of autumn made him feel sad! The wind is bleak; the grass and trees are falling and withering. It's desolate, like walking alone in the distance, or climbing mountains to send people back. The sky is clear and cool. The water is clear and calm. It's sad that autumn cold invades people. It's sad and melancholy that everything in the world is discarding the old and replacing with the new. It's frustrating that poor people are always ignored. Lonely, stay in other places without friends; Lonely, only have self-pity. Swallows fly south, and cicadas are lonely and silent. The wild geese are flying south, the chickens are chirping. From night to day, he can't sleep. The cricket's whine in the dark night triggers the love. With the passage of time, he has passed the middle age, but still stay in place.

It fully shows the look of autumn. From the standpoint of Confucian classics in the Han Dynasty, Wang Yi thought that all the scenery had symbolic meanings, such as "heaven is high and air is clear". Wang Yi noted that "it refers that even invisible things can be seen to sigh unwise and fatuous monarch." If leaving aside the previous interpretation of classics and study, from the perspective of the text itself, this paragraph is actually the text in which the author melts the sad feeling of life into the autumn scenery and perfectly expresses it. In Liu Xie's "Wuse", "When the poet was infected by objective things, he associated with all kinds of similar things. He clung to the universe and thought of what he saw and heard." Although in "Book of Songs", it also shows the characteristics of Song Yu's description: the high integration of heart and object, emotion and object, which can also be called "interweaving the spirit in image". The poet first sums up his sadness on autumn, then writes the life sadness caused by "the withering and death of plants", then writes the sense of loneliness under the clear sky, and finally 
writes that time is late and nothing can be achieved. Abstract "sadness" is constantly sought from the scenery.

This kind of emotion has many resonators in later generations. Pan Yue's "Autumn Prosperity Fu" directly quoted words of Song Yu to trigger the sentiment: "Song Yu's said it well: 'sadness, the autumn has formed a cold and gloomy atmosphere. Depression and loneliness, the grass and trees are shaking and falling into decline. The heart is desolate and sorrowful, like going on a long journey, climbing mountains to see off those who are about to return.' Those who return have the memory of missing their partners, those who travel far have the resentment of having the chance to drift, those who are near the water are like the flowing water of Confucius as time flies, and those who climb the mountain are like the king of Qi Jing mourning for the future. Those four kinds of sentimentality make a person heart painful, and people meet one of them will feel uncomfortable. But sad autumn has endless sorrow and nothing can compare with it." ("Wenxuan", Volume 13) Pan Yue's "Fu of Climbing Hulao Mountain", recorded in the seventh volume of "Collection of Arts and Culture" says: "climbing mountains and facing the water, it is the way to express the grief of the ancients, let alone people who leave home, far away." Like Song Yu, he seemed to write autumn but to write his sorrow. Liu Xie said Pan Yue "is skillful in expressing sadness" ("Eulogy Stele"), and his skill of writing sorrow could not be separated from the imitation of Song Yu.

Lu Ji's "Chant of Spring" said: "the four seasons are as touching and sentimental, but spring is even more so." (Collection of Art and Culture, volume 3, fragmentary). Han Yu's "A Feeling of Spring" says: "the heaven divided a year into four seasons, and spring is the most miserable." There seems to be a relationship between them. In fact, they all come from the third chapter of Song Yu's "Jiubian": "God divided a year into four seasons, spring, summer, fall and winter. Only fall makes me feel sad." What follows in the following is the scenery of dew, long night and so on. The description of various things makes this article "gorgeous", and the continuous and strong sadness is a kind of "touched feeling". "Chu Yin" by Li Shangyin: "On the peak of high mountain stands the palace of Li Gong. The riverside in front of the palace, however, sees the east of the twilight river flowing across the sea. In the boundless sky of Chu state, dusk is always full of wind and rain. Song Yu can't help worrying even if he has no worries." All of these can be seen the influence of Song Yu's theme of mourning for autumn.

The most famous description of autumn in Qu Yuan's Ci and Fu is "Jiuge · Madam Xiang": "The daughter of Emperor Yao came to the north sandbank. Her distant insight made me sad. Autumn wind continuously was blowing and rippling Dongting Lake. Yellow leaves were falling. Through the weeds, look into the distance. I have planned for dating last night. Why do birds fall in apples and fish nets on trees? That girl has a crash on that boy but dare not speak." This passage wrote that the daughter of Yao loved Shun. The writer used "sorrow" to express such sadness. In order to set off this sadness, the description of natural scenery was also to render such an emotional atmosphere to readers. Wang Yi wanted to explain the implied meaning with the description of the scenery. He wrote: "the autumn wind is strong. Grass and trees sway, and Xiangshui River ripples, and the leaves fall. The description of scenery is to state the tightened politics of the emperor, and the sorrow of common people and the worries of talents." ${ }^{2}$ Wang Yi intentionally suggested a causal relationship between "autumn wind" and "curl", "Dongting wave" and "under the tree", and then naturally attaches to such a political theme as dynasty politics and public reaction, which will naturally destroy the emotional nature of the original poem. A similar example is "Jiuge - Mountain Ghost": "The thunder rumbled and the rain fell, and the ape's shrill voice chirped through the night." ${ }^{3}$ Wang Yi quoted and explained: "thunder is a symbol of princes, a metaphor for the monarch. The rain is a metaphor for the gathering of crafty sycophants. The ape's sad and shrill voice is a metaphor for the villain's tongue. The mountain wind is a metaphor for political chaos. The rustle of fallen leaves is a metaphor for the horror of the people." The original intention of $\mathrm{Qu}$ Yuan was to increase his "melancholy" feeling with the autumn wind and the rustling wood leaves. This shows that Liu Xie's skill of "description of scenery" was quite mature in $\mathrm{Qu}$ and Song $\mathrm{Yu}$. The description skill of Song $\mathrm{Yu}$ in "sad autumn" obviously comes from "Jiuge" and has been enlarged. Liu Xie's "Bixing" comments on the skill of "description of scenery" in Fu of Han Dynasty: "As for Yang Xiong, Ban $\mathrm{Gu}$, Cao Zhi and Liu Zhen, all the later writers used the method of "comparison" to display their literary talent in describing mountains and rivers." It is thought that there are complex disadvantages in the description of scenery. In fact, compared with Qu Yuan and Song Yu, there obviously has a sense of "complexity", and from the perspective of literary development, this is exactly the development of Song Yu to $\mathrm{Qu}$ Yuan, which has the effect of following the events and increasing the intensity.

\section{SADNESS IN BUMPY LIFE}

The general emotional type of "Jiubian" is sadness, so the writer was sad due to his bumpy life and unhappiness rather than the desolate scenery. The later is the repeated theme of Qu Yuan's "Lisao" and "Jiuzhang". In Song Yu's "Jiubian" the theme is also very prominent.

In the first chapter of "Jiubian", "It's frustrating that poor people are always ignored." Among them, the word "bumpy" has become a common language in later generations. "Wenxindiaolong · Talent" comments on Feng Yan: "Feng Yan was very fond of giving speeches, but he was not successful in the prosperous period of the Eastern Han dynasty. However, when he wrote "Xianzhi Fu", he felt like a clam who had a pearl." Emperor Ming of the Eastern Han Dynasty thought that Feng Yan was not talented so he didn't use him. This word was to express one was frustrated for all one's talent. Zhong Rong's "Shipin - Zhongpin" comments on Guo Pu: "to express their own feelings of frustration, rather than a simple search for immortal poetry." It said Guo

\footnotetext{
Hong Xingzu, "Supplementary Notes to Chu Ci”, Zhonghua Book Company, 1983, page 65.

Hong Xingzu, "Supplementary Notes to Chu Ci", Zhonghua Book Company, 1983, page 81.
} 
Pu's "Youxian Poem" was also to express the frustration of poor scholars.

\section{Chapter five of "Jiubian" says:}

Why is the time and custom so skillful and disobey the rules and abandon the rules! Refuse to ride on that fine horse, but urge the inferior horse to come on the way. There is no such fine horse in the world, and no coachman can drive it. Seeing that the reins were made up of people, the horses leaped far away. Wild ducks eat rice and fresh vegetables, and the Phoenix has to spread its wings. When I insert a square mortise into a round mortise, I know it must conflict. Every bird has a place to live; only the Phoenix has no place to live. I would like to be silent from now on and never forget your kindness to me. When Jiang Taigong was ninety years old, he was honored. Because of his previous experience, he did not agree with others. Where can a good horse find a home? Where can the Phoenix find its home? It's not ancient that the world wind is declining. Nowadays, Xiangma people only know how to choose fat and give up thin. The steeds are all hidden and never appear again. The Phoenix can't fly far away. Birds and animals still know how to be virtuous, how can they question the wise people not to stay elsewhere? Good horses never rush to drive, and the Phoenix is not greedy for food. The monarch is lighter than abandoning and never discerning. How can a sage exert his ambition if he wants to be loyal! Want to be silent from now on, and do not forget your kindness. Sad autumn alone secretly hurt the God, when to rest angry and strong sorrow!

This is the chapter closest to "Lisao" in "Jiubian". The general emotion is attributed to sadness as well as "Lisao". "Lisao" says: "Everyone is racing to meet greed, and the pursuit of demand is never satisfied." Wang Yi said: "those who are in office who have no desire to be incorruptible and are greedy for money. Although they are full of money, they are still insatiably greedy for money." Song Yu's so-called "current fashion" is similar to Qu Yuan's "those" who are greedy and competitive. In Song Yu's works, these "people pursuing current fashion" betrayed the law of benevolence and righteousness and rejected the talents. Song Yu's images of "fine horse" and "phoenix" which are used to describe the special person also come from Qu Yuan: "riding on a fine horse", "let the phoenix fly night and day" ("Lisao"). Different from Qu Yuan, Song Yu incorporated "fine horse" and "Phoenix" into the system of comparison with the rest of the world, showing that bad horses rule, steed was far away. All birds have a place to live, but the phoenix has nowhere to stay alone. The reason for this lies in the fact that "A good horse never asks for a ride rashly, and a phoenix does not eat food at will." The wise people are noble and don't want to mix with the common customs. "They would like to be silent from now on and do dumb, and cannot forget your kindness." Like Qu Yuan, he wandered between advance and retreat. Or it is the description of Qu Yuan's emotion, in which the method of image analogy is similar to "Lisao" and "Jiuzhang".

From "Lisao" to "Jiubian", there is a kind of emotional tradition of poets characterized by "sadness". If you want to trace the origin of this emotional tradition, it is in "300
Poems". Liu Xi recorded in the fifth chapter of "Shigai" in the second volume of "Yigai": "'wind changes' is led by "Baizhou". "Baizhou" and "Lisao" have the same theme. When you read these two works, you should be able to understand their aspirations and circumstance at the same time." Kong Yingda takes "Ying", "Yi" and "Wei" as the first to change the wind. ${ }^{4} \mathrm{Liu} \mathrm{Xi}$ 's record is more specific to the first chapter "Baizhou" in "Bei Feng" and thinks it is the same theme as "Lisao". Through a careful examination of these two articles, there is indeed something in common in their themes. "Preface to Mao's Poems": "'Baizhou' is to those who are upright but are not appreciated." "Lisao" is also to show Qu Yuan's "performance of loyalty but being framed". Qu Yuan wrote "Lisao" when he was extremely upset. In terms of it, "Baizhou" says: "it's hard to get rid of sadness. These black men are so evil." "Lisao" says: "The world is muddy. It's difficult to distinguish good and evil. Bad man covers good because of resentment." "Baizhou" says: "My heart is not a pebble to roll about on." "Lisao" says: "As long as it is what I really love, I will not regret dying for it many times." His spirit of fearing nothing and unyielding echoed the poets of the two times. Therefore, the thirty-seven volume of "Shuoshi Suiyu", by Shen Deqian, says: "Lisao' is a descendant of 'Book of Songs' which is divided into positive part and negative part. 'Lisao' was written in the background of political decline, so it has the voice of depression, but not the voice of peace. To read its words and identify its music, like a child attached to his parents and do not want to leave. 'Lisao' shows that the loyal men rebuked crafty fawns, love the king, worry country, enough to call the human ethics of the acme. Is it too much to respect it as scripture?" It can be said that the theme of "benevolence without appreciation" has long been appeared in "Book of Songs", which became a familiar emotion type of Chinese literati through the writing of Qu Yuan and Song Yu. Among the poets of later generations, Ruan Ji's "Yonghuai" and Chen Zi'ang's "Ganyu" all repeat this kind of emotion, leaving a lot of touching poems.

\section{THE INHERITANCE RELATIONSHIP AND JOINT NAME OF QU YUAN AND SONG YU}

"Records of the Historian - Biography of Qu Yuan and Jia Yi" says: "after the death of Qu Yuan, there were Song $\mathrm{Yu}$, Tang Le and Jing Chai in Chu. All of them were good at literature, by which they were known by people. They all followed the euphemistic writing style of $\mathrm{Qu}$ Yuan, and always dared not advise directly to the monarch." It is pointed out that Song $\mathrm{Yu}$ and others are scholars after $\mathrm{Qu}$ Yuan. Later, all kinds of historical documents called Song $\mathrm{Yu}$ as Qu Yuan's disciple. ${ }^{5}$ Song Yu may not be directly taught by Qu Yuan, and there is no clear record that Song Yu was Qu Yuan's disciple before the Han Dynasty. The saying of "disciple" is obviously out of the imagination of later

"Shijing Chuanshuo Huizuan" (Si Ku Quam Shu), compiled by the court of Qing Dynasty in the fifth Yongzheng Year, records about Kong Yida: "Bei, Yong and Wei have wide scope and have poems as early, so they become leaders."

Refer to "Research Materials of Song Yu" in "Mingyi Tongzhi", compiled by Liu Gang et al. and other relevant literature. Commercial Press, 2015 , page 6 . 
generations, which shows that there is an inheritance relationship between $\mathrm{Qu}$ Yuan and Song $\mathrm{Yu}$, at least in the imagination of later generations. This kind of inheritance relationship can be seen from the analysis of "Jiubian" and other texts. Next, Liu Xie's records will be listed for further understanding.

When discussing the influence and creative features of Chu Ci in "Lisao", Liu Xie pointed out:

Since Wang Bao's "Jiuhuai", many of his works have been copied from "Chu Ci", but the good examples of $\mathrm{Qu}$ Yuan and Song Yu have always lagged behind. Qu Yuan and Song Yu's feelings of resentment and suppression make readers deeply moved by their pain. The parting emotion they told also makes the reader feel sad and unbearable. When they talked about mountains and rivers, people can think of the appearance of rock valleys from the syllables of the articles. When they talked about the seasons, one can see the changes of time in their writings.

First of all, the joint name of "Yu \& Song" is paid attention to. Sima Qian put Song Yu "good at $\mathrm{Ci}$ and $\mathrm{Fu}$ after $\mathrm{Qu}$ Yuan, and didn't put their name together. The joint name of them was first appeared in Shen Yue's "Songshu - Biography of Xie Lingyun". It says: "Since the decline of the Zhou dynasty, there were a number of handsome literati, Qu Yuan, Song Yu first, Jia Yi, Sima Xiangru followed. Their talents were carried forward. The essence of their words is enough to warm the stone, and the profound meanings of the article are quite precious." It can be seen that Song Yu, as a "Literary Jester", has become a first-class writer in the era of "literary consciousness" in the Qi and Liang dynasties. In addition, Song Yu's works were widely loved in the periods of Jin, Song, Qi and Liang, which can be seen from the description of Song Yu's works in "Wenxuan". Therefore, Liu Xie often naturally put "Qu and Song" together: "Sima Xiangru loved reading and studied works of Qu Yuan and Song Yu. A large number of exaggerated gorgeous descriptions become the master heart of $\mathrm{C}$ and Fu." ("Talent") It reflects the general view of that era. "Yibu" quoted from "Tianzifang", the external chapter of "Zhuangzi". Yan Hui said Dao Fuzi was "a person of exceptional ability", and later generations were unable to catch up. It is the same in writing poetry, which can be learned in form but need to be understood in charm. "Excellence of Qu and Song" refers to the non-trace parts of their works that can only be obtained by wonderful understanding, which are hard for ordinary people to trace. The achievements of Qu Yuan's works have been recognized in the world since the Han Dynasty. The saying of "excellence of Qu and Song" was the highly praise of Song Yu. Here, Liu Xie mainly listed three aspects of "stating the feeling of separation", "describing four seasons" and "depicting the landscape". According to the first section of Lin Shu's "Chunjuezhai Essay · Farewell", " 'Shejiang' says: 'I lament that the barbarians in the south do not understand me. Tomorrow morning I will cross the Xiangjiang River. Boarding E'zhu and looking back, bemoaning the autumn and winter wind cold. Let my horse wander on the hills, and park my cart by the woods. Go upstream in a ship with a window, the oars rise in waves. Ships are slow and not easy to advance when encountering the vortex. In the morning, set out from Wuzhu, and in the evening, stayed in Chenyang. If my heart is really straight, even if the destination is remote. When entering into Xupu, I was hesitating and confused where the direction of travel was. The dark forest, where the apes live, is black with dim light. The mountains are steep and dark and rainy. Snows are boundless. Clouds and mist covers the sky. Lament that life has no joy, alone in the mountains. I will not change my ambition to obey the customs; I will be troubled and distressed and embarrassed until I die.' This paragraph, a small space to accommodate the feelings of parting, talks about the landscape, the four season's climate. Autumn water filled the sky. The evening has arrived in Xiangjiang River. From Wuzhu to Chenyang, at first there is no place to stay. Then in the deep forest with apes and lamentable rain and snow, there was one who had left the capital, not only without a place for himself, but even if the heart had a place to settle? He also knows that the state is in decline, and there is no one who can tolerate him. However, he was unwilling to change himself and obey the customs. Before finishing reading the full text of "Shejiang", he just read this paragraph and thought quietly, and then felt a deep sadness." are various, and the most important thing is to improve the ability to express feelings and portray things on the basis of "300 Poems". This is not only realized in the quotation of "Jiubian", but also can be seen in the following discussion.

"Shilei" also says:

It is said that the works of Qu Yuan and Song Yu were written according to "Book of Songs".

Wang Yi's "Preface to Chuci Zhangjiu" says, " 'Lisao' was written on the basis of the spirit of the five classics." People of Han Dynasty said that Qu Yuan wrote Lisao according to "Book of Songs", while Liu Xie extended it to Song Yu. "Lisao" and other works also recorded Yao, Shun, Tang and $\mathrm{Wu}$ like the Confucian classics, but they are not necessarily from the five classics, so the original words of "Shi" and "Shu" cannot be seen in "Lisao". However, Han Fu likes to follow "original words" of the classics, which is the difference between $\mathrm{Chu} \mathrm{Ci}$ writers and Han $\mathrm{Fu}$ writers. $\mathrm{Li}$ Xiang's "Supplementary Notes" to "Wenxindiaolong · Shilei" says: "Xiangru's 'Daren' followed the style of 'Travel Far Away', and Mei Shu' 'Qifa' carried forward the style of 'Lvlan'. Both of them used the words of original works." ${ }^{8}$ In the Han Dynasty, the study of classics was prevalent and literati were used to "words of original works" and also followed the style of "only stating the achievement of former and not setting new meaning". Jia Yi's "Pengniao Fu" says: "The state of $\mathrm{Wu}$ was very strong, but Fu Chai failed... 'Fu Legend' said it is because Fu Yao liked to slave people to work for him and Wu Ding, Emperor of Yin, appreciated him and arrange him

\footnotetext{
6 Lin Shu's "Chujuezhai Essay".

7 Hong Xingzu. Supplementary Notes to Chu Ci, Zhonghua Book Company, 1983, p 49.

$8 \quad$ Notes to Wenxindiaolong. Noted by Huang Shulin, supplemented
} by Li Xiang, proofread by Yang Mingzhao, Zhonghua Book Company, page 476 . 
as prime minister. It's for guidance, not for use. The allusions compress the story of the ancients into a word or a sentence, as if it was said by themselves, but it implies a story. In the study of Qu Yuan's and Song Yu's works, although they talked about many ancient things, they didn't use the original words and sentences, instead of later Cui Jia, Ban Gu, Zhang Heng, Cai Yong and so on, collecting all kinds of historical books of classics, writing the articles in a colorful way, and relying on ancient books to achieve achievements. "Lisao", $\mathrm{Qu}$ Yuan's representative work, is the product of the combination of Chu culture and "Book of Songs". It not only inherits the tradition of "satirizing monarch" in "Book of Songs", but also develops the technique of "metaphor" in a system and achieves great artistic effect. For Song Yu's works, there are also many traces of "Book of Songs". For example, when describing the beauty of the son of his master in Song Yu's "Dengtuzi Haose Fu", "Her eyebrows, skin, waist and teeth are all no beauty. When she smiled, that beauty is more difficult to describe, fascinating all people in Yangcheng." It is obviously that the description of beauty used the description of Zhuang Jiang's beauty in "Book of Songs": "Her hands are like thatch of fine needles. Her skin is white and smooth, like condensation oil. Her neck is like longicorn's larva soft white tender. Her wide forehead is like cicadas. Her slender curved eyebrows are fine. When she smiled, she had charming cheeks and bright black and white eyes." This shows that Song Yu consciously studied "Book of Songs" and used it. In fact, Liu Xie established a model for the skill of "allusion".

"Shixu" also records:

Qu Yuan's poems can be more comparable to the sun and the moon. Song Yu's literary talent is also beautiful as clouds.

One explanation is that $\mathrm{Qu}$ Yuan's Fu often talks about the sun and the moon. For example, "Lisao" records: "the sun and the moon do not stay in a hurry", "the branches of wood were broken off to block the sun" and "Send Wang Shu in front to lead". Song Yu' "Wind Fu" and "Gaotang Fu" likes to describes "clouds". So, they compares their works to the sun, the moon and clouds. In addition, Liu'an, the king of Huainan, wrote that $\mathrm{Qu}$ Yuan had a noble character in his "Lisao Zhuan". He said: "Qu Yuan's ambition can compare with the glory of the moon and the son." "Talent" says:

In the warring states period, the court employed force, but the literati continued to appear. The hundred schools of scholars relied on their thoughts and doctrines, and Qu Yuan and Song $\mathrm{Yu}$ expressed their extraordinary splendor by reference of $\mathrm{Chu} \mathrm{Ci}$.

Liu Xie's "Zhuzi" comments on the articles of these scholars: "words illustrating theories and expressing ideas". $\mathrm{Qu}$ Yuan was also in that era, and their books were good at literary talent. This is also an evaluation of the era of literary consciousness. The literature of Lisao style came into being after "Book of Songs" and rose up before the writers of $\mathrm{Ci}$ and Fu. It is the source of $\mathrm{Ci}$ and Fu. Then he said, "However, its language is very gorgeous and elegant, and it can be the origin of $\mathrm{Ci}$ and Fu." Liu Xie pointed out that the characteristic of Lisao style is the elegant and gorgeous style of $\mathrm{Ci}$ and $\mathrm{Fu}$, which is the principle of $\mathrm{Ci}$ and $\mathrm{Fu}$ creation.
Liu Xie thought that the reason for the emergence of Lisao style is that there were many talents in Chu. In the later part of "Biansao", Liu Xie said that Liu Xie here for the first time put $\mathrm{Qu}$ Yuan and Song Yu in the same position in literary history. He believed that only Song $\mathrm{Yu}$ could catch up with $\mathrm{Qu}$ Yuan, which changed the previous scholars' cognition of Song Yu. Before Liu Xie, Song Yu had been shrouded in Qu Yuan's aura. The previous scholars' evaluation of Qu Yuan agreed that he was not only a poet of noble origin but also a man of great patriotic sentiment. In one hand, they were always influenced by Sima Qian's evaluation of "always dared not advise directly to the monarch". It not only denied Song Yu's personality, but also his works. Therefore, in the eyes of previous critics, Song Yu could not be at the same height as Qu Yuan. From this, it can be seen that Liu Xie's evaluation has a promotion effect on Song $\mathrm{Yu}$.

\section{CONCLUSION}

To sum up, the description skill of "sad autumn" in Song Yu's "Jiubian" is the inheritance and development of Qu Yuan's "Jiuge". The emotional type of "frustrated mood" in "Jiubian" is also the theme repeatedly chanted in Qu Yuan's works, and it constitutes a kind of emotional tradition of poets characterized by "sadness" from "Lisao" to "Jiubian". From this, it can be seen the inheritance relationship between Song Yu and Qu Yuan in writing skills and emotional types. The inheritance relationship between Qu Yuan and Song Yu can be seen from the way that ancient scholars put Qu Yuan and Song $\mathrm{Yu}$ jointly, which was first appeared in "Song Shu - Biography of Xie Lingyun". Later Liu Xie often put their name together and highly praised the works of Song Yu. Liu Xie's evaluation of Song Yu changed the influence of Sima Qian's bad evaluation on Song Yu and his works were also affected by the negative comments. Liu Xie's high evaluation of Song Yu greatly promoted Song Yu.

\section{REFERENCES}

[1] Hong Xingzu. Supplementary Notes to Chu Ci. Zhonghua Book Company, 1983. (in Chinese)

[2] Liu Gang et al. Research on Historical Materials of Song Yu. Commercial Press, 2015. (in Chinese)

[3] Yang Mingzhao. Notes to Wenxindiaolong. Shanghai: Shanghai Ancient Books Publishing House, 1982. (in Chinese)

[4] Wu Guangping. Research on Song Yu. Changsha: Yuelu Publishing House, 2004. (in Chinese)

[5] You Guo'en. Anthology of Chu Ci. Shanghai: Shanghai Literature and Art United Press, 1955. (in Chinese)

[6] Zhang Shaokang. History of Theoretical Criticism of Chinese Literature. Beijing: Peking University Press, 2005. (in Chinese) 